\title{
LA ASTRONOMÍA EN TEXTOS ESCOLARES DE EDUCACIÓN PRIMARIA
}

\author{
GARCÍA BARROS, S., MARTÍNEZ LOSADA, C., MONDELO ALONSO, M. y VEGA MARCOTE, P. \\ Facultade de Ciencias da Educación. Universidade da Coruña. Paseo de Ronda, 47. \\ 15011 La Coruña.
}

\section{SUMMARY}

In this paper we analyse the way in which the study of Astronomy is dealt with in Primary School textbooks. Moreover, we study how the topic should be approached at this school level.

\section{INTRODUCCIÓN}

En el ámbito de la enseñanza de las ciencias, la astronomía es un tema que plantea problemas de enseñanzaaprendizaje. Nadie duda, en la actualidad, de su importancia educativa, en cuanto a su carácter cultural (Lanciano, 1989; Nussbaum, 1990), a su incidencia en el conocimiento del medio y valor práctico en el desenvolvimiento del sujeto en el mismo, a su capacidad para plantear problemas que han de ser resueltos mediante la utilización de actividades científicas, etc. Por tal motivo, se recomienda incluirla ya desde los primeros niveles de enseñanza (Sharp y Moore, 1993). Concretamente en nuestro sistema educativo se contempla el estudio de la astronomía desde la educación infantil hasta la secundaria obligatoria (ver DCB). La educación primaria es una etapa importante, pues en ella los niños, apoyándose en las ideas elementales que han adquirido en la educación infantil, construyen las bases imprescindibles para comprender los aspectos astronómicos más abstractos y explicativos en secundaria obligatoria, llegando incluso a realizarse aquí las oportunas relaciones con la mecánica clásica. En este sentido, debemos indicar que la relación de la astronomía con otras áreas (matemáticas, geografía...) hace de ella una materia claramente interdisciplinar. Esta interdisciplinaridad puede constituir una ventaja para el proceso de aprendizaje cn cuanto que favorece el establecimiento de relaciones conceptuales y, por tanto, el aprendizaje significativo; aunque también puede suponer un inconveniente y conducir a aprendizajes inconexos, meramente memorísticos, si las relaciones entre unidades didácticas no son las pertinentes.

Ni la presencia de la astronomía en la enseñanza obligatoria, ni su carácter interdisciplinar han permitido superar las concepciones e ideas erróneas que habitualmente poseen Ios ciudadanos. Es habitual que los estudiantes: sostengan concepciones alternativas respecto a las causas del cambio de estaciones en las zonas templadas de la Tierra (Baxter, 1989; Ojala, 1992; Schoon, 1992; Camino, 1995; De Manuel, 1995); interpreten las fases de la luna como eclipses (Camino, 1995; García Barros et al., 1996); tengan una visión geocéntrica đel universo (Alfonso et al., 1995); coloquen estrellas dentro del sistema solar; desconozcan que las estrellas, a excepción de la Polar, cambian de posición a lo largo de la noche; asocien la presencia de la Luna exclusivamente al cielo nocturno...

Esta situación tiene diversas causas: 
a) La propia dificultad cognitiva de este tema y de otros relacionados con él (optica, luz..., geometría...) (Nussbaum, 1986).

b) Ia ausencia de evidencias claras y perceptibles que avalen el movimiento terrestre.

c) El lenguaje, ya que no podemos olvidar que la revolución copernicana no «revolucionó» el lenguaje cotidiano, que sigue siendo geocéntrico y expresa lo directamente observable (Lanciano, 1989).

d) La metodología utilizada habitualmente en el aula, caracterizada por el excesivo enciclopedismo (Arribas de Costa y Rivière, 1989) y por la falta de observaciones directas del cielo, no siempre estimuladas por los libros de texto empleados (Lanciano, 1989).

e) Ia deficiente formación del profesorado, tanto desde cl punto de vista teórico como didáctico. Los profesores en ejercicio o en formación sostienen concepciones alternativas semejantes incluso a las de los niños y adolescentes (Ojala, 1992; Camino, 1995; García Barros et al., 1996), con el consiguiente efecto multiplicador en la población escolar.

f) El tipo de vida, cada vez más urbano que no facilita las observaciones del cielo, tan cotidianas y necesarias en otro tiempo.

Sin embargo, y a pesar del desconocimiento científico que hemos señalado, en la población actual existe un interés creciente por los temas astronómicos. Prueba de ello son las habituales noticias que aparecen en los medios de comunicación sobre eclipses, cometas...; Ia creciente apertura de planetarios y museos en nuestras ciudades; el propio interés mostrado por los niños y adolescentes sobre la carrera espacial...

Todo lo indicado hasta ahora justifica la necesidad de que se produzca un cambio en los planteamientos didácticos de la astronomía. La generalización de tal cambio depende, en cierta medida, de que se modifique el tratamiento que se viene haciendo en los textos escolares. En

Tabla I

Aspectos analizados en los libros de texto.

\begin{tabular}{|c|c|}
\hline CONTENIDOS CONCEPTUALES & ASPECTOS OBJETO DE ANÁLISIS \\
\hline $\begin{array}{l}\text { ¿Sc ajustan los contenidos conceptuales a los } \\
\text { jefinidos en el DCB? }\end{array}$ & $\begin{array}{l}\text { Dado que el DCB es normativo, consideramos aquí solamente la posibilidad de } \\
\text { que los contenidos sean más ampítos. }\end{array}$ \\
\hline Sccucnciación de conteniobos & $\begin{array}{l}\text { Número de veces que se trata la astronomia a lo largo de la ctapa. } \\
\text { Coherencia de la secuenciación conceptual con las recomendaciones teoricamen- } \\
\text { te fundamentadas. }\end{array}$ \\
\hline Contextualizacion & $\begin{array}{l}\text { Existencia de relaciones entre los contenidos astronomicos y aspectos cotidianos, } \\
\text { próximos e interesantes para los alumnos. }\end{array}$ \\
\hline Referencias históricas y culturales & $\begin{array}{l}\text { Referencias a cientificos relevantes, al signifjeado mitológico que nuestros } \\
\text { antepasados dieron a las constelaciones... }\end{array}$ \\
\hline Observación & $\begin{array}{l}\text { Existencia de alguna actividad en la que se realicen observaciones directas del } \\
\text { cielo o se propongan observaciones personales. }\end{array}$ \\
\hline Realización de orientaciones & $\begin{array}{l}\text { Propuesta de actividades encaminadas a desamollar estrategias de orientación en } \\
\text { el espacio noctumo y diurno, utilizando puntos de referencia. }\end{array}$ \\
\hline Manejo de modelos y aparatos & $\begin{array}{l}\text { Fimpleo de la brújula, prismáticos... Utilización de modelos sencillos o realización } \\
\text { de dramatizaciones para simular los movimientos de la Tierra y la Luna. }\end{array}$ \\
\hline $\begin{array}{l}\text { Utilización de mapas y otros sistemas } \\
\text { de representación }\end{array}$ & Orientación en mapas y planos sencillos. \\
\hline Formulacion de hipótesis & $\begin{array}{l}\text { Introducción de preguntas abiertas y divergentes qus permitan a los alumnos } \\
\text { definir sus ideas e interpretaciones sobre los fenómenos astronómicos. }\end{array}$ \\
\hline Análisis de datos y apreciacion de regulatidades & $\begin{array}{l}\text { lnclusión de actividades en las que se anałicen datos para apreciar regularidades: } \\
\text { diarias, mensuales, anuales. }\end{array}$ \\
\hline
\end{tabular}


este sentido es imprescindible destacar que, si bien los libros de texto no son el único material cmpleado por los profesores, su presencia e influencia sigue siendo importante en la enseñanza obligatoria. Además aunque los contenidos que en ellos se incluyen deben ajustarse a las orientaciones del DCB, la apertura del nuevo currículo ofrece múltiples posibilidades sobre orientaciones metodológicas, de secuenciación... la falta de experiencia del profesorado en este último aspecto (hasta el momento no se había enfrentado a secuenciaciones tan amplias) va a incrementar el protagonismo de los textos escolares, que tendrún influencia, no sólo en cada curso concreto, como en los años preceđentes, sino también a lo largo de toda la etapa. En este sentido es necesario destacar que cada editorial opta por una determinada secuenciación y organización de contenidos que va a condicionar, hasta cierto punto, la elección de una editorial concreta para varios cursos.

Por todo ello, en este trabajo analizamos cómo se plantea la astronomía en los libros de texto correspondientes a la educación primaria. Además, basándonos en el análisis realizado y en la bibliografía consultada, aportamos unas consideraciones generales sobre cómo debería ser dicho planteamiento en el citado nivel educativo.

\section{METODOLOGÍA}

Utilizamos los textos de educación primaria de cinco editoriales de amplia difusión en nuestro pais que denominamos A, B, C, D, y E; son, por tanto, treinta libros los analizados.

En este estudio nos centramos en el análisis cualitativo de los contenidos conceptuales y procedimentales. Excluimos, en esta ocasión, el relativo a jos contenidos de ámbito actitudinal, por exceder los límites de este trabajo.

En Ia tabla I se resumen los aspectos analizados, enumerándose en primer lugar los correspondientes a los contenidos conceptuales.

- Contenidos incluidos: Se analiza si los textos recogen únicamente los contenidos conceptuales propuestos en eI DCB de educación primaria o se amplían con conceptos y explicaciones recomendadas para posteriores etapas.

- Secuenciación de los contenidos a lo largo de los tres ciclos de primaria: Se contempla en qué ciclos se incluye la astronomía y se estudia si las secuenciaciones planteadas por las editoriales se corresponden con las propuestas por varios documentos (MEC, 1992; Laliena y Sánchez Iniesta, 1992; Gabinete de Estudio para la reforma educativa, 1992), que resumimos a continuación:

Primer ciclo: Tratamiento observacional, vivencial y descriptivo de los astros (Sol, Luna y sus fases, estrellas), efectos del Sol (produce luz y calor).
Segundo ciclo: Estudio de regularidades (cambios de posición del Sol a lo largo del đf́a, variación de la forma de la Luna y la duración del ciclo lunar, variaciones de las horas de luz y temperatura a lo largo del año), introducción a la orientación espacial.

Tercer ciclo: Los movimientos terrestres y lunares y sus consecuencias (paso del tiempo, fases, eclipses), estrellas, planetas.

- Contextualización: Relación de Ios contenidos con aspectos vivenciales próximos y útiles para los niños. Nos referimos a aspectos espacio-temporales (puntos cardinales, medida del tiempo) y a otros asociados a ellos (costumbres, fiestas...), conquista del espacio...

- Referencias históricas y culturales: Visión dinámica y cultural del conocimiento científico que ofrecen los textos (referencias a descubrimientos realizados por científicos relevantes, significado mitológico que otras culturas dieron a constelaciones y planetas...).

Los contenidos procedimentales se analizan de forma indirecta, infiriéndolos de las actividades propuestas en los textos y en los cuadernos de campo. Nos centramos especialmente, según se indica a continuación, en el análisis de aquellos procedimientos que están específicamente relacionados con el estudio de la astronomía.

- Observación del cielo: Presencia de actividades que promueven la observación directa del cielo (posiciones del Sol a lo largo de la jornada, formas y posiciones de la luna, características de las estrellas, las constelaciones y los planetas, sus posiciones...) y la observación indirecta (sugerencias del texto para que el alumno realice observaciones personales de los aspectos antes indicados).

- Realización de orientaciones: Desarrollo de habilidades de orientación, tomando determinados puntos de referencia (posición del Sol, sombra en distintos momentos de la jornada, la estrella polar). Se consideran tanto las actividades que se desarrollan en el medio exterior como las simulaciones de aula.

- Manejo de modelos y aparatos: Instrumentos que faciliten la observación y la orientación (prismáticos, brújula). Utilización de modelos reales y sencillos (sistemas de esferas) o realización de dramatizaciones que permitan simular los movimientos de la Tierra y la Luna e interpretar sus consecuencias; es decir, que permitan relacionar los movimientos simulados con los fenómenos observables.

- Utilización de mapas y planos: Trabajos de orientación en planos y mapas de puntos concretos (edificio, calle...).

- Formulación de hipótesis: Inclusión de preguntas y actividades orientadas a la detección de las ideas e interpretaciones del alumno. Nos fijamos sobre todo en aquéllas, recogidas en la bibliografía, que influyen especialmente en el aprendizaje de conceptos incluidos en 
educación primaria (forma de la Tierra, nuestra posición en el planeta, dirección de caída de los cuerpos) (Nussbaum, 1989).

- Análisis de datos y apreciación de regularidades: Presencia de actividades en las que el alumno tenga que recoger o analizar datos que permitan apreciar e inferir regularidades diarias (posiciones del Sol, cambio del tamaño o dirección de las sombras, horario del orto o del ocaso solar y lunar), mensuales (formas lunares) y anuales (horas de Iuz, temperatura).

El análisis de textos fue realizado de forma independiente por dos miembros de nuestro equipo, discutiéndose los aspectos discrepantes.

\section{RESULTADOS}

Observamos que los textos de las editoriales de educación primaria analizadas ajustan los contenidos conceptuales a los propuestos por el DCB, es decir, todos ellos tratan aspectos relativos al cielo diurno y nocturno, movimientos terrestres, el ciclo lunar, planetas, estrellas y estaciones del año. Solamente en una de ellas (B) se aprecio un incremento de estos contenidos, abordándose en $6^{\circ}$ curso la explicación teórica del cambio estacional, donde se destaca la variación anual de la inclinación de los rayos solares. Las demás editoriales, aunque también incluyen los cambios estacionales, 10 hacen sólo desđe el punto de vista fenomenológico, circunscribiendo la traslación terrestre exclusivamente a la medida del año.

Respecto a la presencia de la astronomía en los distintos ciclos, debemos indicar que todas la editoriales proponen su estudio en más de un ciclo, aunque dicha presencia es mayor en el segundo. Sólo la editorial $C$ incluye este tema en todos los cursos mientras gue la $\mathrm{E}$ Io excluye del primer ciclo (Tabla II). Además, Ios textos analizados siguen en general las propuestas de secuenciación de contenidos conceptuales recogidas en documentos tomados como punto de referencia de este análisis (ver Metodología). Así, las editoriales plantean un tratamiento observacional, descriptivo y vivencial de los astros y sus efectos en el primer ciclo y el estudio de regularidades astronómicas diarias, mensuales y antuales en el segundo. Sin embargo, todas la editoriles sin excepción incluyen en el segundo ciclo los movimientos terrestres, e incluso dos de ellas (B, E) la trastación Iunar, en contra de las orientaciones de secuenciación anteriormente mencionadas que sugieren su tratamiento en el tercer ciclo de primaria. En este sentido, debemos indicar que en los textos correspondientes a este último ciclo se vuelve a tratar el modelo heliocéntrico.

Con relación a la contextualización de los contenidos observamos que es más evidente en el primer ciclo. Todas las editoriales relacionan los sencillos contenidos astronómicos con el paso del tiempo (día y noche, las estaciones) y con los hábitos y costumbres de Ios seres humanos. En el $2^{\circ}$ y 3 r. ciclo también se asocian los fenómenos astronómicos al paso del tiempo, e incluso un texto lo utiliza como hilo conductor de una unidad didáctica $\left(\mathrm{E}, 6^{\circ}\right.$ curso). Las referencias a las fiestas relacionadas con eventos astronómicos (solsticios y equinoccios) se recogen en una de las editoriales (C, $2^{\circ}$ ciclo). Por otra parte, las editoriales D y E en el segundo ciclo tratan aspectos relativos a la conquista del espacio (naves espaciales, astronautas...).

Las referencias a personajes históricos en los textos son todavía escasas en estos niveles, solamente encontramos una relativa a Galileo (C, $5^{\circ}$ curso). Respecto a las relaciones culturales y mitológicas, señalaremos que únicamente en dos editoriales (C y D) se menciona lo que representaban algunas constelaciones conocidas, como

Tabla II

Presencia de la astronomía en los distintos ciclos de primaria.

\begin{tabular}{|c|c|c|c|c|c|}
\hline \multicolumn{6}{|c|}{ Cursos en que se incluyen el estudio de la astronomía en las distintas editoriales. } \\
\hline Ciclo & Editorial A & Editorial B & Editorial C & Editorial D & Editorial E \\
\hline$I^{0}$ & $2^{\circ}$ & $2^{\circ}$ & $1^{\circ}, 2^{\circ}$ & $1^{\circ}$ & . \\
\hline $2^{\circ}$ & $3^{\circ}, 4^{\circ *}$ & $3^{\circ}, 4^{\circ}$ & $3^{\circ}, 4^{\circ}$ & $3^{\circ}, 4^{\circ *}$ & $4^{\circ}$ \\
\hline $3^{\circ}$ & $5^{\circ \neq}$ & $6^{\circ}$ & $5^{\circ *}, 6^{\circ}$ & $5^{\circ}$ & $6^{\circ}$ \\
\hline
\end{tabular}


Casiopea y Orión, para las civilizaciones antiguas. Otra editorial se reficre al origen romano de nuestro calendario ( $\mathrm{E}, 6^{\circ}$ curso), y al origen de los nombres de los días de la semana (E, $4^{\circ}$ curso).

A través del análisis de contenidos procedimentales, recogidos en la tabla $11 \mathrm{I}$, hemos observado que:

- Existen pocas actividades dirigidas a la observación directa del cielo. Únicamente la editorial $C$ propone en el primer ciclo la observación del Sol -tomando las debidas precauciones- y de las primeras estrellas del creptísculo. En las demás se invita a los alumnos a comprobar las formas de constelaciones, brillo de ciertos planetas... mediante la realización de observaciones personales. Esto lo hemos considerado como observación indirecta. (Segundos ciclos de todas las editoriales y tercer ciclo de la editorial $C$ ).

- Aprender a orientarse uno mismo en el espacio o en el plano es un procedimiento claramente asociado al estudio de la astronomía. Si bien prácticamente todas las eđitoriales, en algún momento, incluyen datos o hechos relativos a los puntos cardinales y a su relación con las posiciones de los astros (la Polar señala el norte, el Sol nace por el este...), la presencia de actividades específicas encaminadas a que el alumno se oriente, dentro 0 fuera del aula, tomando determinados puntos de referencia es escasa, detectándose solamente en dos editoriales (B, 3er curso; C, $3^{\circ}, 4^{\circ}$ y $6^{\circ}$ cursos).

- La utilización de instrumentos de observación y medida, como prismáticos, brújula o reloj de sol, no está generalizada. Únicamente en la editorial C se propone su empleo ya desde el primer ciclo, mientras que las demás no recogen actividades en este sentido. Por otra parte, Ias editoriales B, C y E plantean Ia realización de dramatizaciones o el uso de modelos sencillos, para simular los movimientos terrestres o lunares, en vez de circunscribirse exclusivamente a los clásicos dibujos y esquemas. Concretamente las editoriales $\mathrm{B}$ y $\mathrm{C}$ en el segundo ciclo presentan actividades dirigidas a simular el día y la noche con un globo terráqueo y una linterna; sin embargo, se limitan exclusivamente a este aspecto y no promueven la interpretación por parte del alumno del movimiento aparente del Sol, es decir, que éste establezca las

Presencia de contenidos procedimentales asociados a la enseñanza-aprendizaje de la astronomía, en los textos de las editoriales utilizadas.

\begin{tabular}{|c|c|c|c|c|c|}
\hline PROCEDIMIENTO & Editorial A & Editorial $\mathrm{B}$ & Editorial C & Editorial D & Editorial E \\
\hline $\begin{array}{l}\text { Observación } \\
\text { directa } \\
\text { o indirecta }\end{array}$ & $\begin{array}{l}\text { Indirecta } \\
\left(2^{\circ} \text { ciclo }\right)\end{array}$ & $\begin{array}{l}\text { Indirecta } \\
\left(2^{\circ} \text { ciclo }\right)\end{array}$ & $\begin{array}{l}\text { Directa } \\
\left(1^{\circ} \text { ciclo }\right) \\
\text { Indirecta } \\
\left(2^{\circ} \text { y } 3 \text { er ciclo }\right)\end{array}$ & $\begin{array}{l}\text { Indirceta } \\
\left(2^{\circ} \text { ciclo }\right)\end{array}$ & $\begin{array}{l}\text { Indirecta } \\
\left(2^{\circ} \text { ciclo }\right)\end{array}$ \\
\hline $\begin{array}{l}\text { Realización } \\
\text { de orientaciones } \\
\text { en el espacio } \\
\text { o el el plano }\end{array}$ & - & $2^{\circ}$ cicio & $2^{\circ}$ y 3 er ciclo & - & - \\
\hline $\begin{array}{l}\text { Utilización } \\
\text { de aparatos } \\
\text { y modelos } \\
\text { sencillos }\end{array}$ & - & $\begin{array}{l}\text { Modelos } \\
\text { sencillos } \\
\left(2^{\circ} \text { ciclo }\right)\end{array}$ & $\begin{array}{l}\text { Prismáticos, cristales } \\
\text { ahumados (1er ciclo), } \\
\text { brújula, modelos } \\
\text { y dramatizaciones } \\
\left(2^{\circ} \text { y } 3 \text { er ciclo }\right)\end{array}$ & - & $\begin{array}{l}\text { Dramatizacio- } \\
\text { nes }\left(2^{\circ} \text { ciclo }\right)\end{array}$ \\
\hline $\begin{array}{l}\text { Utilización } \\
\text { de sistemass } \\
\text { de representación }\end{array}$ & $\begin{array}{l}\text { Mapas } \\
\text { (3er cicio) }\end{array}$ & - & $\begin{array}{l}\text { Mapas planos } \\
\left(2^{\circ} \text { ciclo }\right)\end{array}$ & & $\begin{array}{l}\text { Grandes } \\
\text { distancias } \\
\left(2^{\circ} \text { ciclo }\right)\end{array}$ \\
\hline $\begin{array}{l}\text { Propuesta } \\
\text { de preguntas } \\
\text { y cuestiones }\end{array}$ & - & - & $2^{\circ}$ ciclo & $2^{\circ}$ ciclo & $2^{\circ}$ y 3 er ciclo \\
\hline Análisis de datos & 3er ciclo & - & $1^{\circ}, 2^{\circ}, 3 \mathrm{er}$ ciclo & Ier ciclo & $2^{\circ}$ ciclo \\
\hline
\end{tabular}


relaciones oportunas entre las posiciones Sol o Tierra simuladas en el modelo y las posiciones adoptadas por el Sol en el cielo a lo largo de la jornada (en nuestro hemisferio, este-sur-oeste).

- Las editoriales A y C, en $6^{\circ}$ curso y en $3^{\circ}$ y $4^{\circ}$ respectivamente, desarrollan procedimientos de representación utilizando mapas y planos. En el libro de $4^{\circ}$ curso de la editorial E se propone a los estudiantes que representen a escala tamaños y distancias de los planetas del sistema solar.

- Tres editoriales (C, D, E) incluyen preguntas abiertas para que el alumno exponga sus ideas. Tales cuestiones son, por ejemplo: ¿Por qué el Sol no se puede ver de noche? (C/3er curso). ¿Qué significa que un eclipse de Sol sea parcial? (D $/ 5^{\circ}$ curso). ¿Cómo se orientaban los navegantes antes de emplear la brújula? (D/5 curso). ¿Se mueven los planetas? ¿Por qué? (E/4 $4^{\circ}$ curso). Suponemos que estas cuestiones constituyen puntos de reftexión donde el profesor debe intervenir. En la editorial D este tipo de preguntas tiene un carácter complementario; es decir, no se percibe su integración en el texto. No se han observado cuestiones o actividades encaminadas a detectar ideas previas relativas a la forma esférica de la Tierra, nuestra posición en ella o a la dirección del campo gravitatorio terrestre.

- Prácticamente todas las editoriales incluyen alguna actividad que, mediante el análisis de datos, bien recogidos por el atumno en periódicos... o aportados por el propio texto, permite apreciar ciertas regularidades típicas de los cambios estacionales (horas de luz, temperatura), de las fases lunares (formas, duración)...

Finalmente debemos indicar que todos los libros analizados plantean actividades orientadas a relacionar conceptos, a facilitar la memorización de datos y definiciones, a utilizar adecuadamente el vocabulario... Este tipo de actividades tiene una especial presencia en los textos de las editoriales B y D. Además, la editorial E $\left(2^{\circ}\right.$ ciclo) hace especial énfasis en el manejo aritmético de los grandes números (tamaños y distancias).

\section{CONCLUSIONES. DISCUSIÓN}

El análisis de textos realizado parece indicar que: a) se sobrevaloran Ios aspectos teórico-conceptuales, apreciándose una «sobrecarga» conceptual especialmente en los correspondientes al $2^{\circ}$ ciclo de educacion primaria; b) se hace todavía poco hincapié en contenidos procedimentales como la observación directa del cielo, la realización de orientaciones en el espacio o la utilización de modelos sencillos que faciliten la comprensión teórica de los movimientos terrestres. Por el contrario, otros procedimientos menos específicos tienen una presencia más generalizada.

Con relación a los contenidos conceptuales, resulta llamativo observar que todas la editoriales incluyen los movimientos terrestres y sus consecuencias en el segun- do ciclo ( $3^{\circ}$ y $4^{\circ}$ curso), aunque varias propuestas de secuenciación recomienden retrasarlos al tercer ciclo (Gabinete de Estudio para a Reforma Educativa, 1992; Laliena y Sánchez Iniesta, 1992; MEC, 1992). Posiblemente la causa de esta situación sea simplemente la «inercia»; siempre se trabajó la rotación y la traslación terrestre y lunares en estos cursos. También resulta curioso que en el texto de $6^{\circ}$ curso de una editorial se expliquen las causas del cambio estacional, cuando este tema encierra serias dificultades de aprendizaje y sobre él sostienen ideas erróneas estudiantes de secundaria c incluso universitarios (Baxter, 1989; Camino, 1995; De Manuel, 1995; Ojala, 1992; Schoon, 1992).

Nos podemos preguntar ahora si es realmente inadecuado explicar el modelo heliocéntrico en el segundo ciclo de primaria. A nuestro juicio no se puede centrar la discusión en principios maximalistas. La idoneidad de introducir o no los movimientos reales de la Tíerra depende, en gran medida, como ocurre con otros temas del mismo nivel de abstracción, de "cómo se explican», «en detrimento de qué otros conteniđos importantes se abordan", "qué aspectos previos se tienen en cuenta para explicarlos»...

En este sentido debemos destacar que para enseñar a los niños que la Tierra se mueve es imprescindible analizar previamente si tienen claro el carácter esférico de nuestro planeta, su posición en él..., pues suelen mantener concepciones alternativas al respecto (Nussbaum, 1989). No debemos olvidar que los niños tienen serias dificultades para comprender que la Tierra está moviéndose en el espacio (Klein, 1982; Nussbaum, 1989). Fsta detección de concepciones se omite sistemáticamente cn todos los libros de texto analizados, to que dificulta, sin duda, el aprendizaje significativo.

Además, la introducción temprana del modelo heliocéntrico se hace generalmente en detrimento de otros contenidos más importantes para los niños de educación primaria. Nos referimos, tanto a los contenidos procedimentales de ámbito intelectual que inciden positivamente en la conceptualización como a otros específicamente asociados a la astronomía que también favorecen la comprensión de conceptos y se contemplan en el currículo oficial (DCB). Dada su importancia haremos a continuación un comentario de los mismos.

La observación directa del cielo es uno de los procedimientos esenciales en el estudio de la astronomía, que no siempre se trata con la amplitud que se merece en los textos escolares. Éstos, salvo excepciones, se limitan a invitar al alumno a realizar observaciones personales $\mathrm{e}$ independientes. La observación, además de permitir la visión tridimensional, la recogida de datos y favorecer el aprendizaje, resulta motivadora para los niños (Lanciano, 1989). No debemos olvidar que la enseñanza tradicional, muy centrada en lo conceptual y abstracto, desestimó lo evidente, lo vivencial y lo cotidiano, que son los aspectos que tienen mayor valor educativo para el nivel al que dedicamos este trabajo. Además es necesario recordar que en la enseñanza de las ciencias es objetivo prioritario que el alumno sepa interpretar cien- 
tíficamente los fenómenos naturales. En cste caso resulta irrelevante que un niño. «sepa» el movimiento de rotación terrestre si «no sabe» explicar con él la variación de la posición del Sol en el cielo a lo largo de la jornada y la sucesión de los días y las noches.

Otro procedimiento importante es la orientación espacial; sin embargo, las actividades diseñadas para que el alumno llegue a desarrollar habilidades de orientación en el cspacio y en el plano son todavía insuficientes en los textos analizados. Éstas, aun siendo útiles para cualquier ciudadano, están poco extendidas en la población, circunscribiéndose en la actualidad, casi exclusivamente, a ciertos colectivos (marineros, agricultores, aficionados a la astronomía).

Asimismo, en la enseñanza de los movimientos terrestres es necesario utilizar recursos sencillos (modelos reales, dramatizaciones) (Estalella, 1986; Osborne, 1991). Con ellos los niños pođrán apreciar directamente cómo son esos movimientos, cuáles son algunas de sus consecuencias para nosotros... Debemos señalar que los textos analizados, no siempre proponen explícitamente actividades en las que se simuten los citados movimientos y se interpreten los fenómenos observables a través de los mismos.

Tambićn es importante la recogida de datos, su análisis, la obtención de conclusiones... Este aspecto está recogido en mayor o menor medida en los textos utilizados, pues el tema de astronomía es especialmente adecuado para analizar datos y apreciar regularidades (fases lunares, variaciones regulares a lo largo del día, año...).

Con relación al desarrollo de procedimientos debemos señalar, además, que en la mayoría de los libros de texto analizados se priorizan especialmente aquellas actividades en las que se trabajan procedimientos de índole general: relacionar, describir, dibujar, buscar información adicional, utilizar procedimientos aritméticos.... Sin negar la importancia de los mismos, creemos que urge la realización de otro tipo de actividades que favorezcan realmente la familiarización con los procedimientos más específicos antes mencionados. Proponemos, por tanto, dedicar mayor espacio a cstos últimos en las unidades didácticas de astronomía orientadas a Ia educación primaria.

Por otra parte consideramos que es necesario dar un giro a los contenidos conceptuales y superar su exclusiva referencia a las teorías científicas. Los niños de esta edad pueden empezar a comprender e interesarse por ciertos aspectos históricos y culturales asociados a la astronomía, nos referimos al significado mitológico de ciertas constelaciones, planetas, a las fiestas que conmemoran solsticios y equinoccios, a las relaciones lingiísticas que existen entre los días de la semana y el nombre de ciertos planetas, el Sol y la Luna, tanto en castellano como en otros idiomas. Estos aspectos, aunque se detectaron en algunos de los textos no constituyen realmente la norma.

Con relación a la secuenciación de contenidos debemos indicar que, si bien las editoriales contemplan la astrono- mía en más de un ciclo de la educación primaria, hemos apreciado diferencias en cuanto a su tratamiento. AIgunas hacen un estudio continuado en los distintos cursos, mientras que otras optan por concentrar dicho estudio en cursos concretos. Dada la larga duración de esta etapa y los cambios psicoevolutivos de los niños a lo largo de la misma, consideramos más idóneo el tratamiento een espiral» de este tema. Este tratamiento del currículo favorece que los conocimientos se construyan y reconstruyan a lo largo de varios cursos (Bruner, 1968). Además como manifiestan Coll y otros (1992), los conceptos a diferencia de los hechos y datos, no se aprenden de una vez, sino que se profundiza en ellos progresivamente.

Finalmente debemos señalar que en astronomía, al igual que en otros temas de ciencias de la naturaleza, se propone la utilización de estrategias y actividades de cambio conceptual (Osborne, 199 l; García Barros et al., 1995) con la consiguiente detección de concepciones previas. Si bien alguna de las editoriales plantea cuestiones abiertas, éstas tienen a nuestro juicio un valor discutible, pues no suelen constituir el inicio de una discusión para exponer ideas ni para apreciar si el alumno es capaz de aplicar los conocimientos en nuevas situaciones.

\section{CONSIDERACIONES FINALES}

A modo de resumen debemos schnalar que la enseñanza de los temas relacionados con la astronomía en Ia educación primaria han de contemplar los siguientes aspectos:

- Los contenidos deben guardar un equilibrio, de tal forma que no se prioricen sólo los conceptuales «clásicos» relativos a los movimientos reales de la Ticra, la Luna y los planetas, en detrimento de aquéllos deI ámbito procedimental. Nos referimos, sobre todo, a los procedimientos específicamente relacionados con la astronomía (observación del cielo diurno y nocturno, desarollo de estratcgias de orientación en el espacio y en el plano, inferencia de regularidades, interpretación de fenómeno observables mediante modelos...).

- Es necesario adecuar los contenidos conceptuales a los intereses, necesidades técnicas y culturales de los estudiantes, haciendo especial hincapié en los fenómenos observables y en los aspectos astronómicos relacionados con las nociones espacio-temporales, tan importantes a desarrollar en educación primaria.

- Se deben adaptar los contenidos a las capacidades intelectuales de los niños y evitar la introducción excesivamente temprana de los modelos teóricos.

- Dado que la comprensión de la teoría heliocéntrica no es evidente y cncierra importantes dificultades para los alumnos de educación primaria, es necesario que los planteamientos didácticos contemplen: a) la relación entre los movimicntos reales de los astros y los aparen- 
tes; b) las ideas e interpretaciones de los niños; c) la utilización de modelos manejables, dramatizaciones... que permitan, no sólo evidenciar los movimientos reales, sino también que los alumnos pongan a prueba sus propias ideas, facilitándose así el aprendizaje significativo.
- I a enseñanza de la astronomía en la educación primaria debe responder a un tratamiento «espiral» a lo largo de los distintos ciclos; esto resultará sencillo, sobre todo si tenemos en cuenta su carácter interdisciplinar, que facilita su introducción en distintos temas y centros de interés.

\section{REFERENCIAS BIBLIOGRÁFICAS}

ALFONSO, R.,BAZOGONZÁLEZ, C., LÓPEZ HERNÁNDEZ, M., MACAU, M.D. y RODRIGLUEL PALMERO, M.L. (1995). Una aproximación a las representaciones del alumnado sobre el universo. Enseñanza de las Ciencias, 13(3), pp. 327-335.

ARRIBAS DECOSTA, A. y RIVIERE, V. (1989). La Astronomía en ta enseñanza obligatoria. Enseñanza de las Ciencias, 7(2), pp. 201-205.

BAXTER, J. (1989). Children's understanding of familiar astronomical events. International Journal of Science Education, 11, pp. 502-513.

BRINER, I. (1968). Hacia una teoría de la instrucción. México: ATHEA.

CAMINO, N. (1995). Ideas previas y cambio conceptual en astronomía. Un estudio con maestros de primaria sobre el día y la noche, las estaciones y las fases de la luna. Enseñanza de las Ciencias, 13(1), pp. 81-96.

COLL, C., POZO, J.I., SARABIA, B., VALLS, E. (1992). Los contenidos de la reforma. Madrid: Aula XXI, Santiliana.

DE MANUEI, J. (1995). ¿Por qué hay veranos e inviernos? Representaciones de estudiantes (12-18) y de futuros maestros sobre algunos aspectos del modelo Sol-Tierra. Enseñanza de las Ciencias, 13(2), pp. 227-236.

ESTALELLA, R. (1986). Astronomía en la enseñanza básica y media. Cuadernos de Pedagogia, 136, pp. 8-11.

GABINETEDEESTUDIOPARAIAREFORMA EDUCATIVA (1992). Educación Primaria. Desenvolvemento Curricular. Santiago de Compostela: Consellería de Educación e Ordenación Universitaria.

GARCÍABARROS,S.,MONIDLO,M.yMARTÍNEZLOSADA C. (1995) ¿Qué vemos en el cielo? Una introducción a la enseñanza de la astronomía. Aula Material , 44.
GARCÍA BARROS, S., MARTÍNEZ LOSADA, C. y MONDELO, M. (1996). Ia astronomíl en la formación de profesores. Alambique (en prensa).

KLEIN, C.A. (1982). Earth and Sun: A cross-cuitural study. Science Education , 65(1), pp. 95-101

I.ANCIANO, N. (1989). Ver y hablar como Tolomeo y pensar como Copérnico. Enseñanza de las Ciencias, 7(2), pp. 173-182.

LALIENA, I. y SÁNCHEZ, INIESTA, T. (1992). Propuesta $d{ }^{2}$ secuencia. Conocimiento del medio. Madrid: MEC. Escucla Española.

MEC. 1992. Primaria. Madrid: MEC.

NUSSBAUM, J. (1986). Students' perception of Astronomical concepts. Proceedings of the Girep Conference Copenthagen, en Martínez Sebastià, B. (1995). Investigación Didáctica en astronomía: Una selección bibliográfica. Enseñanza de las Ciencias, 13(3), pp. 387-389.

NUSSBAUM, J. (1989). La Tierra como cuerpo cósmico, en Driver, R., Guesne, E. y Tiberghien, A. Ideas cientificts en la infancia y la adolescencia. Madrid: MEC, Morata.

NUSSBAUM, J. (1990). Astronomy teaching: challenges and problems. IVth International Conference on Teaching Astronomy. Barcelona.

OJALA, J. (1992). The third planet. International Journal of Science Education, 14(2) pp. 191-200.

OSBORNE, R. (1991). Approaches to the teaching of AT16the Earth in space: issues, problems and resources. School Science Review, 72(260) pp. 7-15.

SCHOON, K.J. (1992). Students'alternative conceptions of Earth an space. Journal of Geological Haducation, 40, pp 209-214.

SHARP, J. y MOORE, K. (1993). Constructivist learnings: the Earth and space sciences-implications for curriculum design at stages I and 2. Teaching Earth Science, 18(4), pp. $130-134$. 